\title{
Selective Strengthening of Conditioned Behaviors That Occur during Periods of Amphetamine Exposure
}

\author{
Xun Wan', John M Holden', Kevin G Lynch ${ }^{2}$ and Laura L Peoples*,1,3 \\ 'Department of Psychology, University of Pennsylvania, Philadelphia, PA, USA; '² Department of Psychiatry, University of Pennsylvania School of \\ Medicine, Philadelphia, PA, USA; ${ }^{3}$ Neuroscience Graduate Group, University of Pennsylvania School of Medicine, Philadelphia, PA, USA
}

\begin{abstract}
Exposure to psychomotor stimulants, during conditioning sessions, can lead to a persistent increase in the strength of conditioned behaviors and the effects of conditioned stimuli, which can be detected in subsequent drug-free periods. It is possible that the effects are selective for the behaviors and stimuli conditioned during drug exposure. The present study was designed to test this prediction. Animals were trained to discriminate two sets of stimuli. For each set, lever pressing during the presentation of one stimulus ( + ) was reinforced and responding during the presentation of the other stimulus $(\mathrm{S}-$ ) had no programmed consequences. Following an initial acquisition phase, training with one set of stimuli continued during sessions of amphetamine exposure, whereas training with the second set continued during saline exposure (20 intermixed sessions). The findings of subsequent drug-free choice tests showed that the drug history selectively enhanced the propensity of animals to engage in the drug-assigned behavior relative to the saline-assigned behavior. This change in behavior was evident in S+, but not $\mathrm{S}$ - trials and was potentially mediated by an acute effect of amphetamine on stimulus conditioning. The findings provide novel evidence that the facilitative effects of coincident conditioning and acute psychomotor stimulant exposure can be selective for the stimuli and behaviors conditioned during the drug exposure. These findings are relevant to hypotheses regarding the etiology of drug addiction.

Neuropsychopharmacology (2007) 32, I346- 1357. doi: I0.1038/sj.npp. I 301250; published online I3 December 2006
\end{abstract}

Keywords: amphetamine; dopamine; drug addiction; learning; choice

\section{INTRODUCTION}

Administration of psychomotor stimulants before Pavlovian and instrumental conditioning sessions can enhance the rate of conditioning. Drug exposure during conditioning can also increase the strength of the conditioned behaviors and the effects of the conditioned stimuli during subsequent drug-free test sessions (eg Kulkarni, 1968; Krivanek and McGaugh, 1969; Glick, 1971; Killcross et al, 1997; Harmer and Phillips, 1998, 1999; Robbins, 1975, 1978; Robbins et al, 1983). One interpretation of these observations is that a history of psychomotor stimulant exposure facilitates future motivated behaviors, generally, and independent of whether those behaviors are conditioned in close temporal association with acute drug actions. An alternative interpretation is that the facilitative effects of psychomotor stimulants is selective for behaviors and stimuli conditioned during the period of acute drug exposure. Differentiating between these interpretations is relevant to understanding potential therapeutic effects of psychomotor stimulants, but may also

*Correspondence: Dr LL Peoples, Department of Psychology, University of Pennsylvania, 3720 Walnut St, Philadelphia, PA 19104, USA, Tel: + I 215898 4253, Fax: + I 2158987301 ,

E-mail: Ipeoples@psych.upenn.edu

Received 28 April 2006; revised 22 September 2006; accepted 26 September 2006 be important for identifying mechanisms that mediate the contribution of the drugs to neuropsychiatric disorders such as drug addiction.

There is considerable evidence that a history of amphetamine exposure can facilitate the future acquisition and expression of both conditioned and unconditioned motivated behaviors, and moreover, that this effect can occur in the absence of any history of coincidence between the facilitated behavior and drug exposure. For example, a history of psychomotor stimulant exposure can enhance the rate at which animals subsequently acquire a novel Pavlovian-conditioned behavior under drug-free conditions (eg Taylor and Jentsch, 2001; Nocjar and Panksepp, 2002; Olausson et al, 2003; Levens and Akins, 2004). Moreover, a history of psychomotor stimulant exposure can enhance the strength of conditioned behaviors and the effects of conditioned stimuli on behavior in subsequent drug-free periods. Specifically, a prior history of psychomotor stimulant exposure can enhance the energizing and reinforcing effects of conditioned stimuli on instrumental behavior, strengthen subsequent approach and instrumental behaviors directed toward primary rewards, and enhance consummatory behaviors directed toward primary rewards (Barr et al, 1999; Fiorino and Phillips, 1999; Taylor and Horger, 1999; Wyvell and Berridge, 2001). In all of these examples, the facilitated behaviors, and the stimuli and rewards that 
showed an enhanced influence on behavior, occurred only under drug-free conditions, and sometimes weeks after the last acute administration of drug. These data are consistent with the conclusion that a history of psychomotor stimulant exposure can have a general effect on the acquisition and expression of future motivated behaviors.

Based on these findings, it is plausible that the effects of pre- and post-session injections are not specific for behaviors and stimuli conditioned during acute drug exposure. Nevertheless, there is some evidence supportive of the hypothesis that coincident conditioning and acute drug exposure can have a selective effect on behaviors and stimuli conditioned during drug exposure. One prediction of the hypothesis is that the facilitative effects observed in association with pre-session injections depend on the cooccurrence of conditioning and acute drug actions. Consistent with this prediction, there are at least two studies that have shown that the facilitative effects of pre-session injections are not observed if the acute drug exposure and conditioning are temporally dissociated (Krivanek and McGaugh, 1969; Robbins, 1978). Another prediction of the hypothesis is that drug exposure in association with conditioning sessions will cause a differential strengthening of either or both conditioned behaviors and conditioned stimuli, within individual animals. More specifically, for individual animals, behaviors conditioned during periods of acute drug actions should be enhanced relative to similar behaviors conditioned during drug-free periods. The present study was designed to conduct a first test of this prediction.

In two experiments, animals were trained to discriminate two sets of $\mathrm{S}+$ and $\mathrm{S}-$ stimuli. After a criterion of discrimination accuracy was attained for both discriminations, training with one set of stimuli was continued during periods of amphetamine exposure, whereas training with the second set of stimuli was continued during periods of saline exposure. The relative strengths of the drug- and saline-assigned conditioned behaviors were characterized using a choice test procedure in which the $S+$ conditioned during drug exposure and the $\mathrm{S}+$ conditioned during saline exposure were presented simultaneously and response manipulanda associated with both conditions were available (Figure 1). The results of the choice tests in conjunction with previous reports of more general effects of amphetamine exposure on reward-related behaviors are consistent with the conclusion that psychomotor stimulants have multiple effects on conditioned behavior, some of which are selective for behaviors that occur during periods of drug exposure and others that are not.

\section{EXPERIMENT 1 MATERIALS AND METHODS}

\section{Subjects}

Subjects were seven male Long-Evans rats (Charles River, Wilmington, MA, $300-325 \mathrm{~g}$ at the start of the study). Animals were housed individually in a temperature- and ventilation-controlled environment under a reversed $12 \mathrm{~h}$ light/dark cycle (lights off at 0800 hours). Rats had access to water ad libitum and were fed $20-25 \mathrm{~g}$ of food each day to maintain body weight at approximately $350 \mathrm{~g}$. Subjects maintained good health throughout the experiment. All animal care and protocols were in accordance with the Guide for the Care and Use of Laboratory Animals published by the US Public Health Service and approved by the Animal Care and Use Committee of the University of Pennsylvania.

\section{Apparatus}

Behavioral sessions were conducted in operant chambers $(30 \times 30 \times 40 \mathrm{~cm})$ purchased from Med Associates Inc. (St Albans, VT). Each chamber was housed in a soundattenuating outer box that contained a red house light and a ventilation fan. Two retractable levers were located on a single wall, $2.5 \mathrm{~cm}$ from the floor and on either side of a centrally located reward trough. On the opposite wall, two

\section{Baseline}

Treatment

Test

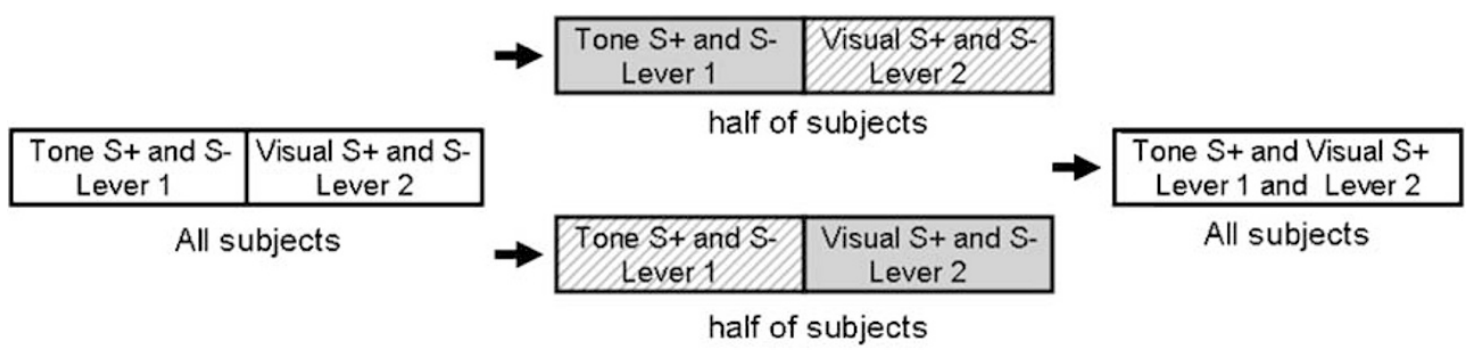

Injection Type

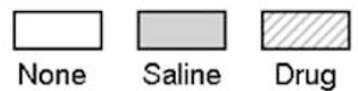

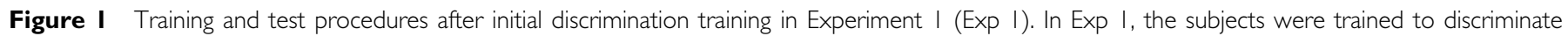

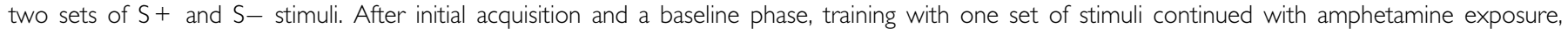

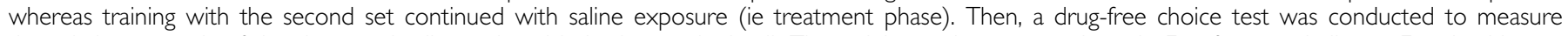

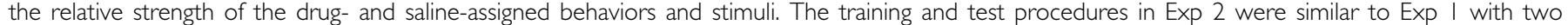

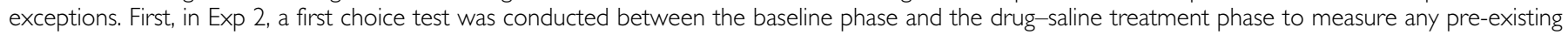
bias in lever preference. Second, in choice tests of Exp 2, S- trials were added in which the saline-assigned S- and the drug-assigned S- were presented in a paired way. 
stimulus lights $(2.5 \mathrm{~cm}$ in diameter) were located $6.5 \mathrm{~cm}$ from the floor and $12 \mathrm{~cm}$ to the left and right of the chamber midline. Two speakers were placed on the chamber ceiling. The chamber was additionally equipped with a clicker device at the top, a cue light $(1 \mathrm{~cm}$ in diameter) inside the trough, and a white noise generator. All experimental events were controlled and recorded using hardware and software purchased from Med Associates Inc.

\section{Behavioral Training}

Discrimination training. Animals were trained to respond on each of the two response levers on a VI- $15 \mathrm{~s}$ schedule of reinforcement (ie $0.25 \mathrm{ml}$ of a combined solution of $3.0 \%$ glucose and $0.125 \%$ saccharin). Reward delivery was signaled by illumination of the cue light inside the trough ( $3.5 \mathrm{~s}$ in duration) and operation of the clicker device (three clicks).

Thereafter, each subject was trained to discriminate between a set of auditory stimuli (a $4.9 \mathrm{kHz}$ constant tone $v s$ a $2.9 \mathrm{kHz}, 1.2$ pulse/s intermittent tone) and a set of visual stimuli (constant illumination of one stimulus light $v s$ intermittent illumination of the other light, 1 pulse/s). For each set of stimuli, one stimulus was designated as the $\mathrm{S}+$ and the other was designated as the $\mathrm{S}-$, counterbalanced across subjects. The response lever associated with a particular discrimination was fixed and assigned in a counterbalanced manner across subjects. Animals were trained on only one of the two discriminations per day.

All discrimination training sessions began with illumination of the red house light and onset of the white noise. Both the red light and the white noise remained on for the duration of the session. Each session included $15 \mathrm{~S}+$ trials, $15 \mathrm{~S}-$ trials, seven $\mathrm{S}+$ probe trials, and seven $\mathrm{S}-$ probe trials. Each trial was $30 \mathrm{~s}$ in duration and separated by an inter-trial-interval (ITI) of $90 \mathrm{~s}$. Trials were scheduled in blocks containing two S+ trials, two S- trials, one S+ probe trial, and one $S-$ probe trial, which were presented in a random order. During an $S+$ trial, lever presses were reinforced according to the VI-15 s schedule. Lever presses during an $\mathrm{S}$ - trial had no programmed consequence. Probe trials were identical to regular trials except that no reward was delivered, and thus provided a sample of responding that was free of any confound introduced by reward consumption, which occurred during regular $\mathrm{S}+$ trials. Discrimination training sessions were scheduled so that each block of two sessions included one auditory session and one visual session. Within each block of two sessions, the order of the two discriminations was randomized. Discrimination accuracy was calculated as a ratio between responses during $S+$ probe trials and the sum of responses during all $\mathrm{S}+$ and $\mathrm{S}-$ probe trials. When accuracy for each discrimination reached $80 \%$ in three of five consecutive sessions, subjects began the pretreatment baseline phase.

Baseline. The baseline phase consisted of 10 sessions, five auditory discrimination sessions and five visual discrimination sessions. On the last 2 days of training on the auditory and visual discriminations, a saline injection (i.p.) was administered to habituate subjects to the injection procedure. These sessions were then followed by a drug-saline treatment phase.

\section{Drug-saline treatment phase.}

Assignment of discriminations to the drug and saline treatments: For each subject, one of the discriminations was assigned to the drug treatment and the other was assigned to the saline treatment. This assignment was counterbalanced across subjects on the basis of the following: (1) number of training sessions completed before the discrimination criterion was attained, (2) discrimination accuracy during the baseline phase, (3) response rates in $\mathrm{S}+$ probe trials during the baseline phase, and (4) location of the response lever, so that a similar number of subjects would respond on the left and right levers during the drug sessions.

The drug-saline treatment: During a 20-day period, subjects were administered an injection of either saline or d-amphetamine sulfate solution $(0.25 \mathrm{mg} / \mathrm{kg}$, i.p. $) 10 \mathrm{~min}$ before the start of each discrimination session. The training sessions for the discrimination assigned to the drug treatment were consistently preceded by an amphetamine injection (10 sessions) and the training sessions for the discrimination assigned to the saline treatment were consistently preceded by a saline injection (10 sessions). In each block of two sessions, one drug session and one saline session were administered in a random order. The amphetamine dose equaled one that was previously observed to enhance conditioned reinforcing effects of stimuli associated with a non-drug reward (Robbins et al, 1983). Following completion of the drug-saline treatment phase, training sessions and all injections were discontinued for 7 days. Thereafter, subjects were exposed to a choice test session under drug-free conditions.

Choice test. At the onset of the choice test, the levers associated with both drug- and saline-assigned discriminations were inserted and remained in the chamber for the duration of the session. The session included two 30-s S+ trials that were separated by a 90 -s ITI. On each trial, the $\mathrm{S}+$ stimuli of the drug-assigned and saline-assigned discriminations were presented simultaneously and subjects were given the opportunity to respond on either lever. Responding on either lever had no programmed consequence.

\section{Data Analysis}

Discrimination training sessions during the baseline and treatment phases. The calculation of response rates as well as discrimination accuracy during all discrimination training sessions was based on probe trials. Preliminary analyses of the response rates showed that there was no effect of session number within either the baseline phase or the drug-saline treatment phase. Moreover, the average response rates during $\mathrm{S}-$ and ITI periods were comparable. Given these findings, session number was included as a repetition within each of the phase $\times$ discrimination $\times$ cue conditions and ITI was dropped from all analyses in order to simplify the design of the ANOVA $(\alpha=0.05)$. Similar methods were used to analyze discrimination accuracy and number of rewards earned per session.

Choice test. Preliminary graphical examination of the data suggested that scores were characterized by non-normal 
distributions. Thus, the data were transformed before conducting statistical analyses. We used the $\log _{10}(x+1)$ transformation, where $x=$ the average response rate of a subject during the choice test. After this transformation, the skewness was reduced to appropriate levels and variance was comparable across different levels of $\log _{10}$ response rates.

\section{RESULTS}

\section{Acquisition}

Average number of training days to criterion was $30.6 \pm 4.9$ days for the discrimination that would be assigned to the drug exposure condition and was $24.7 \pm 2.7$ days for the discrimination that would be assigned to the saline exposure condition. No significant difference was found between the two discriminations $(t(6)=1.01, p>0.3)$.

\section{Baseline and Treatment Phases}

A series of ANOVAs were used to test for differences in behavior either between the baseline and treatment phases, or between the discriminations assigned to drug and saline. The behavioral measures subjected to these ANOVAs included the following: (1) discrimination accuracy, (2) operant response rates, and (3) number of rewards earned per session.

Discrimination accuracy. Discrimination accuracy was comparable between the discriminations assigned to the drug and saline treatments and remained stable across the baseline and treatment phases (Figure 2a). Consistent with this observation, a two-factor ANOVA, with phase (ie baseline $v s$ treatment) and discrimination (ie assigned to drug or saline) as factors, showed that there was no significant effect of either phase $(\mathrm{F}(1,6)=1.56, p>0.2)$ or discrimination $(\mathrm{F}(1,6)=0.28, p>0.6)$. There was also no significant interaction between phase and discrimination $(\mathrm{F}(1,6)=3.73, p>0.1)$.

Response rates during discrimination sessions. Response rates during discrimination sessions varied differentially across the baseline and treatment phases for the two discriminations (Figure 2b). A three-factor ANOVA, with cue (ie $\mathrm{S}+v s \mathrm{~S}-$ ), phase, and discrimination as factors, confirmed that there was a significant interaction among cue, phase, and discrimination $(\mathrm{F}(1,6)=9.29, p<0.05)$, as well as a significant interaction between discrimination and phase $(\mathrm{F}(1,6)=10.3, p<0.05)$. Separate two-factor ANOVAs, with cue and phase as factors, were subsequently applied to response rate data of the two discriminations. These analyses showed that for the discrimination assigned to saline, there was no significant effect of phase $(\mathrm{F}(1,6)=2.28, p>0.05)$, and no significant interaction between phase and cue $(\mathrm{F}(1,6)=2.04, p>0.05)$. Therefore, response rates during the discrimination assigned to the saline condition remained stable. In contrast, for the discrimination assigned to drug, there was an effect of phase $(\mathrm{F}(1,6)=25.89, p<0.01)$ and a significant interaction between phase and cue $(\mathrm{F}(1,6)=29.38, p<0.01)$. Further comparisons showed that the $S-$ response rate during the
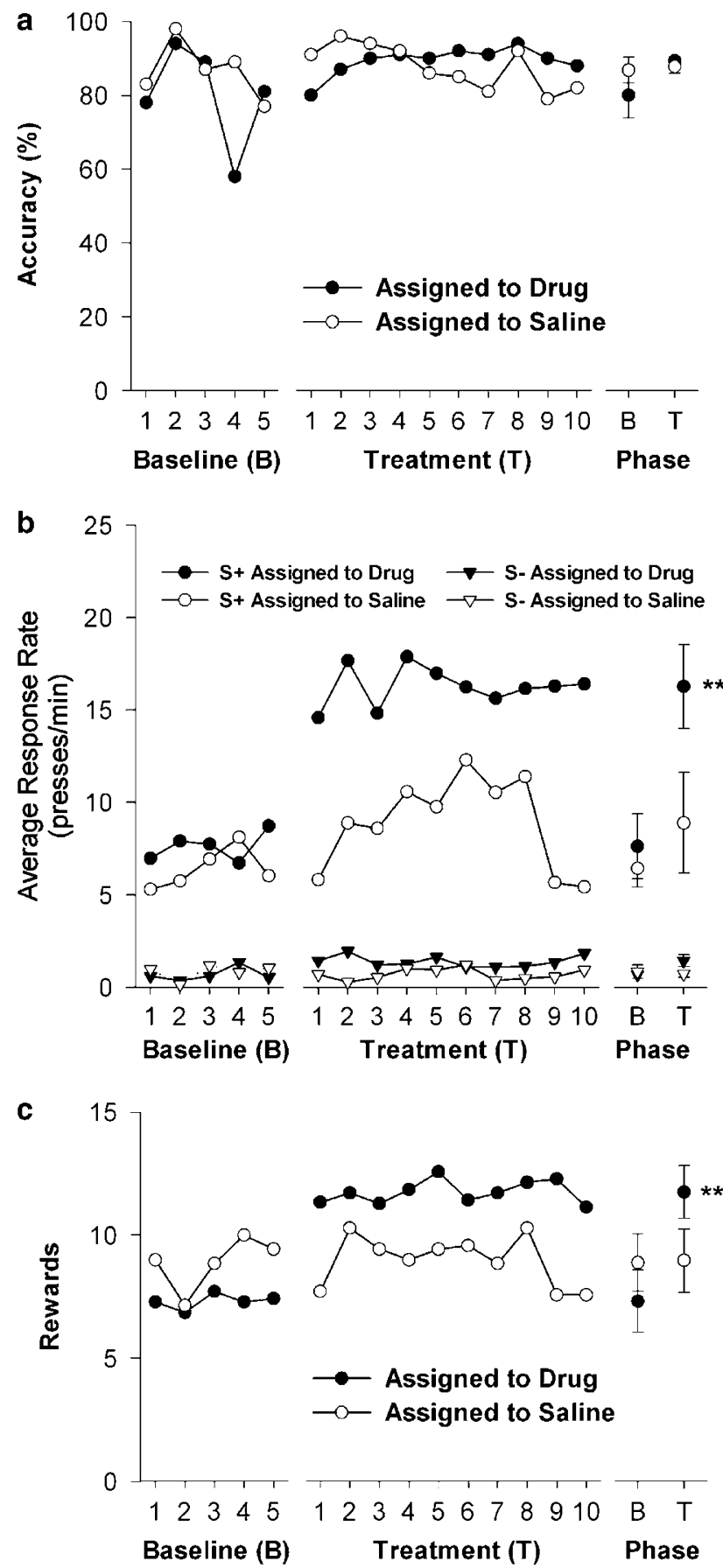

Figure 2 Behavioral measures during the baseline and treatment phases of Exp I. (a) The average discrimination accuracy ratio is plotted for all subjects as a function of session for both the baseline phase and the treatment phase. (b) Average response rates during probe $\mathrm{S}+$ and $\mathrm{S}-$ trials are plotted as a function of session for both the baseline phase and the treatment phase. (c) Average number of rewards earned per session is plotted for all subjects as a function of session for both the baseline phase and the treatment phase. To the right of each plot is shown the average for the baseline phase (B) and for the treatment phase (T), with the standard error of the mean $( \pm$ SEM). Asterisks indicate a significant change from the baseline phase to the treatment phase. ${ }^{*} p<0.01$.

discrimination assigned to drug did not change across the baseline and treatment phases $(t(6)=1.95, p>0.05)$; however, the $S+$ response rate increased significantly 
$(t(6)=5.33, p<0.01)$. Moreover, the $S+$ response rate was comparable for the two discriminations during the baseline phase $(t(6)=0.62, p>0.5)$ but marginally greater for the drug sessions than for the saline sessions during the treatment phase $(t(6)=2.63, p=0.056)$.

Number of rewards earned during discrimination sessions. The number of rewards earned during the two discriminations changed differentially between the baseline and treatment phases (Figure 2c). A two-factor ANOVA, with phase and discrimination as factors, showed that there was a significant interaction between discrimination and phase $(\mathrm{F}(1,6)=24.6, p<0.05)$. Further comparisons showed that for the discrimination assigned to drug, the number of earned rewards increased during the treatment phase relative to the baseline phase $(t(6)=4.43, p<0.01)$. In contrast, for the discrimination assigned to saline, the number of earned rewards did not change between the two phases $(t(6)=0.14$, $p>0.05)$.

Choice test. During the choice test, subjects responded more on the drug-assigned lever than on the saline-assigned lever (Figure 3). Consistent with this observation, a twofactor ANOVA with cue (ie S+ vs ITI) and lever (ie drug$v s$ saline-assigned) as factors showed that there was a significant effect of lever $(\mathrm{F}(1,6)=7.40, p<0.05)$. However, there was no significant interaction between cue and lever $(\mathrm{F}(1,6)=2.88, p>0.1)$. Therefore, the differential responding on the drug- and saline-assigned levers was not selective for the $S+$ trials.

\section{EXPERIMENT 2}

There were aspects of the design and findings of Exp 1 that confounded interpretation of the choice test data of that experiment. Exp 2 was conducted to address these issues.

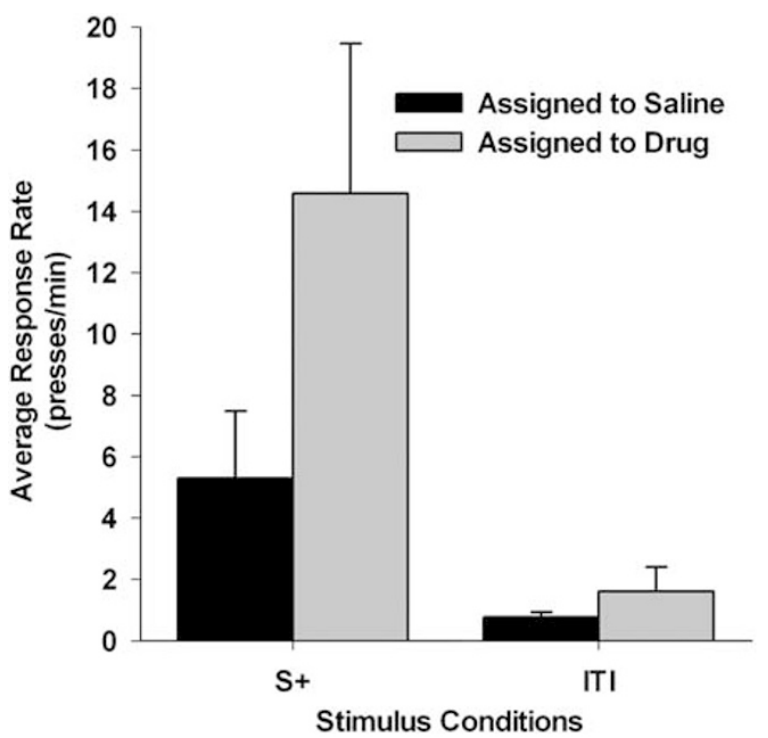

Figure 3 Response rates on the drug- and saline-assigned levers during the choice test of Exp I. Average response rates (+ SEM) on the lever associated with the drug-assigned discrimination and the lever associated with the saline-assigned discrimination are plotted for the $S+$ trials and for the intervening ITls.
In Exp 1, drug exposure during the treatment phase increased response rates and the number of earned rewards during the drug sessions relative to the saline sessions. These observations are consistent with other evidence that acute amphetamine exposure can increase the rate of operant responding and thereby lead to an increase in the number of earned rewards (Robbins et al, 1983). However, the changes in response rates and number of earned rewards confounded interpretation of the choice test data. Specifically, the differential responding during the choice test could be due to the behavioral differences that emerged between the drug and saline sessions during the treatment phase. For example, the increased rates of operant responding during the drug sessions could have conditioned a habit to respond at a faster rate on the drugassigned lever. Additionally, the increased number of rewards earned during the drug sessions could have strengthened the behavior conditioned during drug exposure via normal operant conditioning mechanisms. A number of methodological changes were made in Exp 2 to avoid this difficulty.

Specifically, training procedures were altered to increase baseline response rates to an asymptotic level and thereby to prevent an increase induced by acute drug exposure. These changes included: (1) a decrease in the total number of probe trials from 14 to 6 , which increased the probability of reinforcement and was thus expected to increase response rates, and (2) a modification of food deprivation procedures that was expected to increase motivation during the training sessions (see Materials and methods of Exp 1 and 2). The altered training procedures were expected to eliminate behavioral differences between discriminations during the treatment phase and thus to eliminate these differences as potential contributors to the response pattern during the post-treatment choice test.

In Exp 1, the subject's relative propensity to respond on the drug-assigned and saline-assigned levers, when both levers were available (referred to as a lever preference) was measured only after the amphetamine-saline treatment. In the absence of a pretreatment assessment of lever preference, a contribution of pre-existing response biases to responding during the post-treatment choice test could not be excluded. To better address this issue, in $\operatorname{Exp} 2$, a pretreatment choice test was added to the experimental protocol. The lever preference of animals exhibited during this first test was counterbalanced during the assignment of discriminations to the drug and saline conditions.

Another issue that was addressed in Exp 2 was whether the drug-saline treatment effect can be selective for responding, controlled by a positive conditioned stimulus. In Exp 1, animals showed increased responding on the drug-assigned lever during both the S+ trials and the ITI periods of the choice test. One interpretation of this result is that drug exposure enhances operant responding through a stimulusindependent mechanism. Alternatively, it is also possible that the drug effect is selective for positive conditioned stimuli, which includes not only the S+ stimulus, but also the stimuli that predict reward and that are present during the ITI (eg the response lever and contextual cues). If the latter interpretation were correct, one might expect that there would be greater evidence of stimulus specificity in a direct comparison of $\mathrm{S}+$ and $\mathrm{S}-$ trials, given that the 
presence of the $\mathrm{S}-$ is expected to suppress the excitatory influence of positive conditioned stimuli that might be present during non-S+ periods (Miller and Spear, 1985). To test this possibility, the choice tests were modified to include $\mathrm{S}-$ as well as $\mathrm{S}+$ trials.

Finally, informal observations made during Exp 1 suggested that the drug exposure regimen during the treatment phase sensitized animals to the psychomotor stimulant effects of amphetamine. Data collection and analysis procedures that would allow us to confirm the development of psychomotor sensitization were added to Exp 2. Sensitization has heretofore been observed to be associated with a general increase in behavioral responsiveness to conditioned stimuli and reward (eg Harmer and Phillips, 1998; Taylor and Jentsch, 2001; Wyvell and Berridge, 2001). The observation of both sensitization and a selective enhancement of behavior conditioned during drug exposure in the same animals would be novel evidence that the occurrence of sensitization does not preclude the possibility of additional selective changes in reward-directed behavior.

\section{MATERIALS AND METHODS}

Except for the aforementioned methodological changes, the methods of Exp 2 were similar to those of Exp 1. The procedures are thus described only briefly.

\section{Subjects}

Subjects were 28 male Sprague-Dawley rats (Charles River, Wilmington, MA, 300-325 g at the beginning of the study). Animals were housed in the same manner as in Exp 1. Subjects were allowed to increase body weight to $380 \mathrm{~g}$. Thereafter, animals were restricted to $85 \%$ of that weight throughout the experiment.

\section{Behavioral Training}

Discrimination training and baseline. In Exp 2, animals were again trained to respond on a VI-15 s schedule of reinforcement. The discriminative stimuli were altered relative to $\operatorname{Exp} 1$ to enhance stimulus salience. The auditory stimuli were a $4.9 \mathrm{kHz}$ constant tone and a $2.9 \mathrm{kHz}, 5$ pulse $/ \mathrm{s}$ intermittent tone. The visual stimuli were a constant light and an intermittent light ( 2 pulse/s). The constant light consisted of sustained illumination of four ceiling level stimulus lights, whereas the intermittent light was pulsed illumination of a second house light in the chamber. On each $\mathrm{S}+$ trial, the stimulus was either presented for a maximum of $30 \mathrm{~s}$ or was immediately terminated as the response requirement was fulfilled and a reward was delivered. On other types of trials, the stimulus lasted for fixed $30 \mathrm{~s}$. As in $\operatorname{Exp} 1$, animals were trained until they reached the discrimination accuracy criterion. Thereafter, animals completed an additional 10 days of training in order to obtain baseline measures of behavior.

The first choice test. Upon completion of the baseline phase, a first choice test was conducted before the onset of the drug-saline treatment phase. The procedure of the choice test was modified relative to Exp 1. The session began with an adaptation period of $30 \mathrm{~s}$ before the two levers were extended into the chamber. In addition, two Strials were added to the session as described so that the total number of trials was increased from 2 to 4 . As was the case in Exp 1, during $S+$ trials, the $S+$ stimuli for both discriminations were presented simultaneously for $30 \mathrm{~s}$. Similarly, during S- trials, the S- stimuli for both discriminations were presented simultaneously. The order of $\mathrm{S}_{+}$and $\mathrm{S}-$ trials was always $\mathrm{S}+, \mathrm{S}-, \mathrm{S}+, \mathrm{S}-$, with an ITI period of $60 \mathrm{~s}$.

\section{Drug-saline treatment phase.}

Assignment of discriminations to the drug and saline treatments: After the first choice test, the auditory and visual discriminations were assigned to either the drug or saline treatment. This assignment was counterbalanced across subjects on the basis of the following: (1) number of days required to achieve the discrimination criterion, (2) discrimination accuracy during the baseline phase, (3) response rates in $\mathrm{S}+$ probe trials during the baseline phase, (4) auditory $v s$ visual discrimination, so that a similar number of subjects were exposed to the drug during the auditory and visual discriminations, and (5) location of the response lever. Subjects were additionally counterbalanced on the basis of lever preference exhibited during the first choice test, so that preference for the lever that would be assigned to the drug treatment was close to $50 \%$ (ie $46 \%$ ) for the whole group. Specifically, a 'preferred' lever was defined as the lever on which the greatest number of $S+$ responses was made by the subject. For 23 out of the 28 subjects, $S+$ responses on the preferred lever accounted for more than $70 \%$ of total S+ responses on the two levers.

The drug-saline treatment and the second choice test: After the assignment, subjects completed two additional discrimination training sessions (one for each discrimination) to counteract any extinction effect that might have occurred during the choice test. The drug-saline treatment phase was initiated immediately thereafter. Except for the use of a 15-min, rather than a 10-min presession injection time, the drug-saline treatment phase was similar to that of Exp 1. Following completion of this phase, training sessions and all injections were discontinued for 7 days. Thereafter, a second choice test identical to the first one was conducted.

Locomotor activity: Subject behavior was videotaped on days 1,5 , and 10 of both the saline- and drug-assigned discriminations during the treatment phase. Locomotor activity was measured by scoring each session and counting the total occurrences of certain types of traversals. These traversals were either from one side of a wall to the other, from one side of the chamber to the other, or from one diagonal corner to the other. The number of occurrences was then converted into centimeters traversed.

\section{Data Analysis}

The data in Exp 2 were analyzed using the same methods that were applied to data of Exp 1, except that the choice test data were not transformed because the increased number of subjects reduced the skewness of the raw data. Moreover, animals in Exp 2 were additionally subjected to separate 
analyses based on their lever preference exhibited in the first choice test.

\section{RESULTS}

\section{Acquisition}

Average number of training days to criterion for the two discriminations did not differ significantly $(t(27)=0.91$, $p>0.3)$. Number of training days equaled $24.7 \pm 0.77$ for the discrimination that would be assigned to drug and $25.7 \pm 0.79$ for the discrimination that would be assigned to saline.

\section{Baseline and Treatment Phases}

Discrimination accuracy. Although discrimination accuracy increased between the baseline and treatment phases, it remained comparable for the two discriminations throughout the experiment (Figure 4a). A two-factor ANOVA, with phase and discrimination as factors, showed that there was a significant effect of phase $(\mathrm{F}(1,27)=30.66, p<0.001)$, but no significant effect of discrimination $(\mathrm{F}(1,27)=0.004$, $p>0.9)$ and no significant interaction between phase and discrimination $(\mathrm{F}(1,27)=1.57, p>0.2)$.

Response rates during discrimination sessions. Both $\mathrm{S}+$ and $\mathrm{S}$ - response rates varied across the baseline and treatment phases; however, response rates remained comparable for the two discriminations throughout the experiment (Figure 4b). A three-factor ANOVA with cue, phase, and discrimination as factors showed that there was a significant interaction between cue and phase $(\mathrm{F}(1,27)=19.53$, $p<0.001)$ but no other significant interactions. Further comparisons showed that the $S+$ response rate during the treatment phase was significantly greater than that during the baseline phase $(t(27)=2.88, p<0.01)$. By contrast, the $S-$ response rate was decreased during the treatment phase $(t(27)=-3.70, p<0.001)$. The differential changes in the $\mathrm{S}+$ and $S-$ response rates were consistent with the betweenphase increase observed in discrimination accuracy.

Number of rewards earned per session. During both the baseline and treatment phases, the number of rewards earned per session was near the fixed maximum of 15 for both discriminations (Figure 4c). A two-factor ANOVA with phase and discrimination as factors showed that there was no significant effect of either discrimination $(\mathrm{F}(1$, $27)=2.23, p>0.1)$ or phase $(\mathrm{F}(1,27)=1.32, p>0.2)$. There was also no significant interaction between discrimination and phase $(\mathrm{F}(1,27)=0.07, p>0.8)$.

Locomotor activity. During the treatment phase, locomotor activity was greater during the drug sessions than during the saline sessions. Moreover, across days of the treatment phase, locomotor activity during the drug sessions increased progressively; whereas, locomotor activity during the saline sessions remained stable (Figure 5). Consistent with these observations, a two-factor ANOVA, with discrimination and day (ie days 1,5 , and 10 of the treatment phase) as factors, showed that there was a significant effect of both discrimination $(F(1,26)=153.86$,
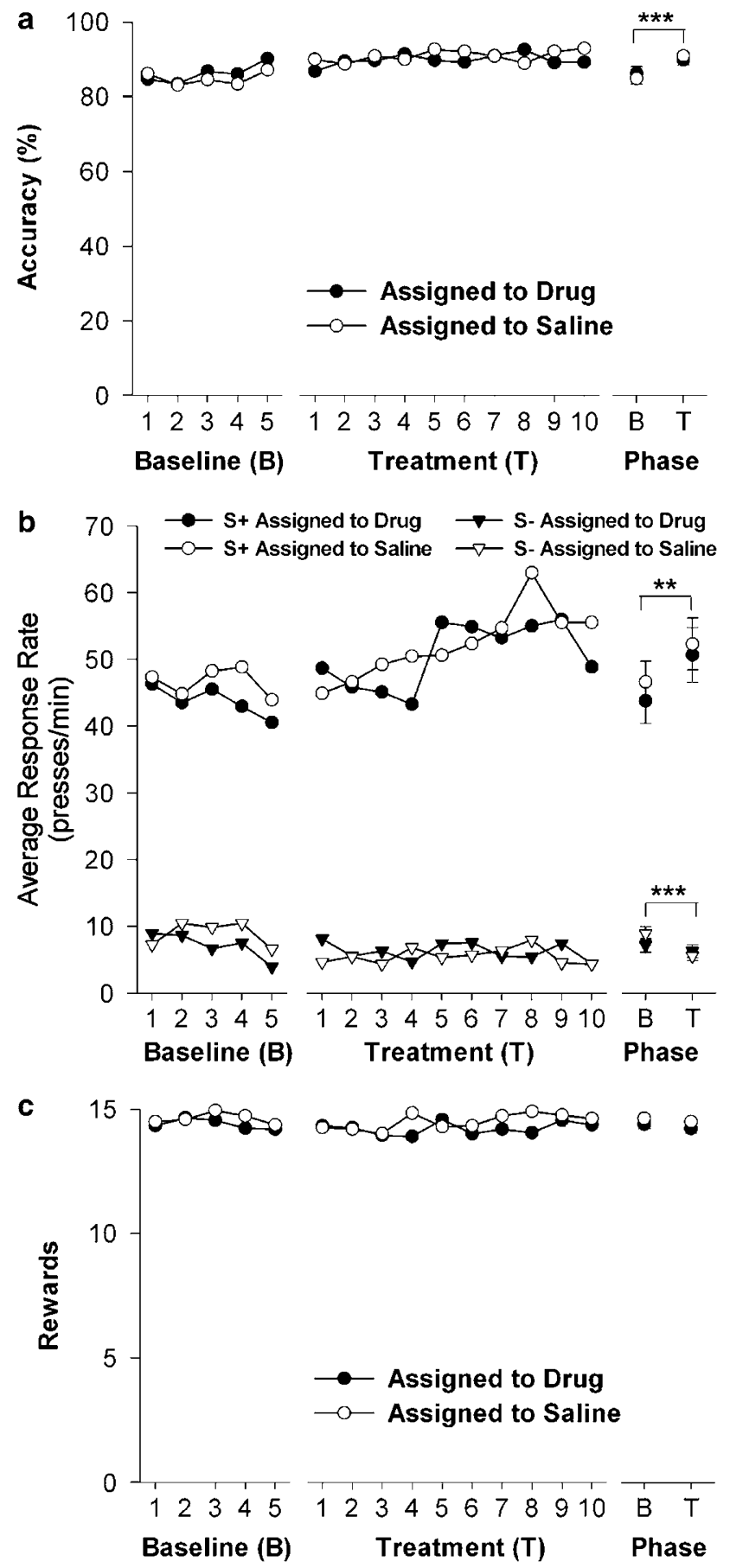

Figure 4 Behavioral measures during the baseline and treatment phases of Exp 2. (a) The average discrimination accuracy ratio is plotted for al subjects as a function of session for both the baseline phase and the treatment phase. (b) Average response rates during probe $\mathrm{S}+$ and $\mathrm{S}-$ trials are plotted as a function of session for both the baseline phase and the treatment phase. (c) Average number of rewards earned per session is plotted for all subjects as a function of session for both the baseline phase and the treatment phase. To the right of each plot is shown the average for the baseline phase $(B)$ and for the treatment phase $(T)$, with the standard error of the mean $( \pm$ SEM). Asterisks indicate a significant change during the treatment phase compared to the baseline phase. ${ }^{*} p<0.01$; **** $p<0.001$.

$p<0.001)$ and day $(\mathrm{F}(2,52)=32.38, p<0.001)$, as well as a significant interaction between discrimination and day $(\mathrm{F}(2,52)=31.45, p<0.001)$. 


\section{Choice Tests}

All subjects. Response rates on the levers assigned to drug or saline exposure changed differentially between the first and the second choice tests (Figure 6). A three-factor ANOVA with test (ie choice test 1 vs 2), cue, and lever (ie assigned to drug $v s$ assigned to saline) as factors showed that there was no significant three-way interaction among lever, cue, and test $(\mathrm{F}(1,27)=2.37, p>0.1)$ and no significant interaction between lever and cue $(\mathrm{F}(1,27)=0.86, p>0.3)$. However, there was a significant interaction between lever and test $(\mathrm{F}(1,27)=7.14, p<0.05)$. Additional analyses confirmed that during the first test there was no difference in responding on the two levers $(t(27)=0.004, p>0.9)$. By contrast, during the second test, responding on the drug-assigned lever was significantly greater than that on the saline-assigned lever $(t(27)=2.51$, $p<0.05)$.

Subjects segregated by their lever preference in the first choice test. During the first choice test, 15 animals showed a preference for the lever that would be assigned to the saline treatment. Following the treatment, nine of them $(60 \%)$

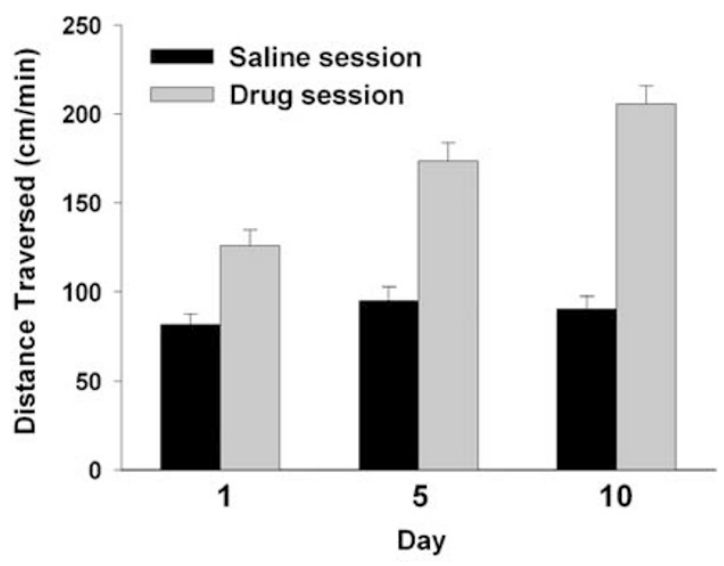

Figure 5 Locomotor activity during the treatment phase of Exp 2. Locomotor activity (cm traversed per min) during the entire drug- and saline-assigned discrimination sessions is shown for 3 days (days I, 5, and 10) of the treatment phase. exhibited a shift in preference to the drug-assigned lever in the second choice test. In contrast, of the 13 animals that originally showed a preference for the lever that would be assigned to drug, only one (7\%) exhibited a shift in preference to the saline-assigned lever during the second choice test. As a result, the percentage of all animals that preferred the lever assigned to drug increased from $46 \%$ during the first test to $75 \%$ during the second test.

These observations were confirmed by ANOVAs applied to average response rates of the two subgroups of subjects (Figure 7). For animals originally biased for the salineassigned lever, a three-factor ANOVA indicated that there was a significant test $\times$ lever $\times$ cue interaction $(F(1,14)=$ $10.96, p<0.01)$, a significant test $\times$ lever interaction $(\mathrm{F}(1,14)=13.06, p<0.01)$, and a significant cue $\times$ lever interaction $(F(1,14)=8.27, p<0.05)$. These results are consistent with the interpretation that the animals originally biased to respond on the lever assigned to saline showed a significant between-test shift, responding more on the lever assigned to drug after the treatment. Moreover, this shift in responding was apparent during the $\mathrm{S}+$ trials but not during the $S-$ trials. Additional analyses showed that this shift in responding was not attributable to differences in either response rates, reward number, or discrimination accuracy between the two discriminations during the treatment phase (data not shown).

On the other hand, animals originally biased for the drugassigned lever showed no significant between-test changes in response rates. A three-way ANOVA indicated that there was no significant effect of test $(\mathrm{F}(1,12)=0.057, p>0.8)$. Additionally, there was no significant interaction among test, lever, and cue $(\mathrm{F}(1,12)=2.17, p>0.1)$ and no significant interaction between test and lever $(F(1,14)=0.003$, $p>0.9)$.

\section{DISCUSSION}

\section{Summary of Major Findings}

In both Exp 1 and 2, animals responded more on the drugassigned lever than on the saline-assigned lever during the post-treatment choice test. In Exp 2, segregation of the
S+ Trials

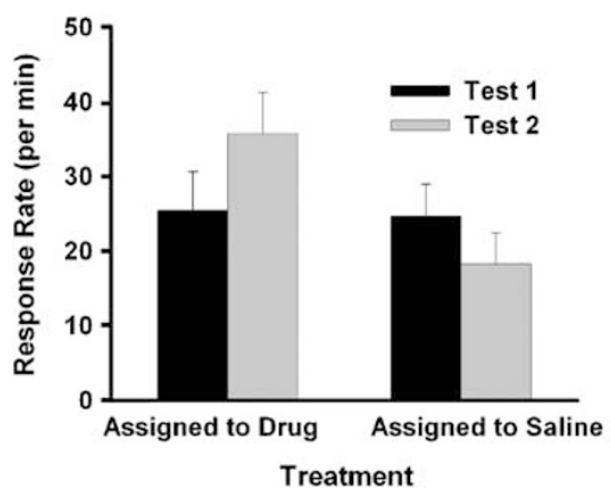

S- Trials

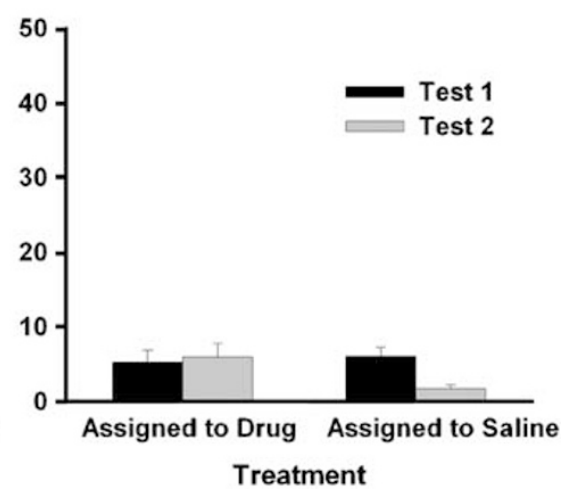

Figure 6 Response rates on the drug- and saline-assigned levers during the choice tests of Exp 2. Average response rates (+ SEM) on the lever associated with the drug-assigned discrimination and the lever associated with the saline-assigned discrimination are plotted for both the first and the second choice tests. Response rates are plotted separately for the $\mathrm{S}+$ trials (left panel) and the $\mathrm{S}$ - trials (right panel). 

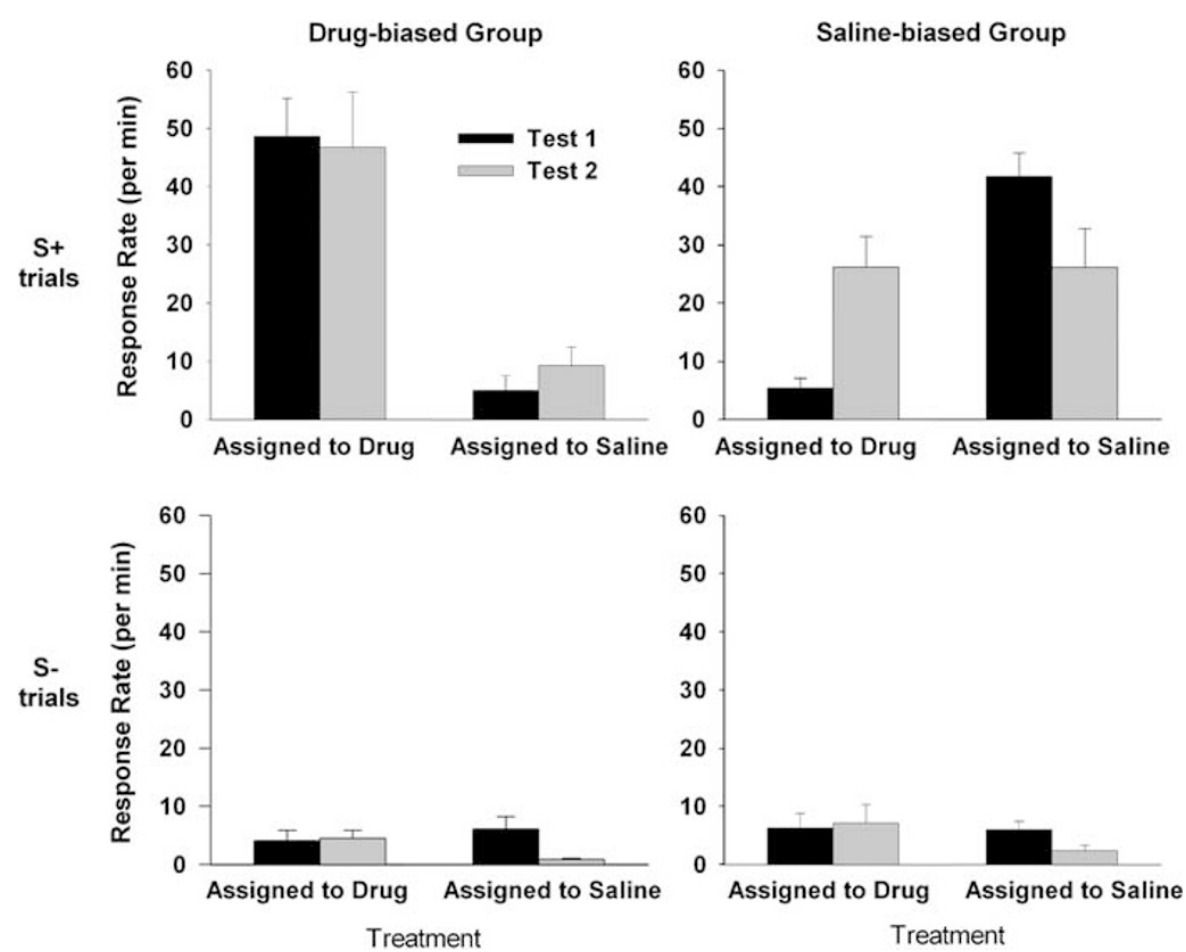

Figure 7 A comparison of between-test changes in response rates between animals that showed different lever preferences during the first choice test. For the two subgroups of animals that exhibited different lever preferences during the first choice test, average response rates (+ SEM) on the lever associated with the drug-assigned discrimination and the lever associated with the saline-assigned discrimination are plotted for both the first and the second choice tests. The left column shows response rates of animals that had a preference for the lever assigned to drug. The right column shows response rates of animals that had a preference for the lever assigned to saline. Within each column, the data are separately plotted for the $S+$ trials (top row) and the $S-$ trials (bottom row).

subjects by their pretreatment lever preference indicated that most of the subjects that showed a pretreatment preference for the saline-assigned lever shifted their lever preference following the treatment. In contrast, animals that showed a pretreatment preference for the drug-assigned lever maintained that preference in the post-treatment choice test. In the animals originally biased to the salineassigned lever, the shift in responding was selective for $\mathrm{S}+$ trials. In both $\operatorname{Exp} 1$ and 2, before the treatment phase, the would-be amphetamine- and saline-assigned discriminations were matched with respect to performance measures (eg discrimination accuracy, response rates) and multiple procedural variables (eg location of response levers, sensory modality). This matching prevented numerous nonpharmacological variables from biasing behavior during the posttreatment choice test. Control analyses also showed that behavior during the choice test was not biased by acute amphetamine-induced changes in discrimination performance. As is discussed below, the present findings are consistent with the interpretation that acute actions of amphetamine strengthened the effects of the drug-assigned $\mathrm{S}+$, such that it controlled instrumental responding more strongly than did the saline-assigned $\mathrm{S}+$.

\section{Acute Effects of Amphetamine That Potentially Contributed to the Post-Treatment Pattern of Choice Behavior}

Acute effects of amphetamine that may explain the present findings include discriminative stimulus effects, state- dependent learning effects, performance effects, and primary reward effects. It is also possible that amphetamine had a direct effect on mechanisms that influence either associative learning or memory consolidation (eg White, 1996; Berke and Hyman, 2000; Everitt et al, 2001; Wolf et al, 2004). Among these effects, some are more likely than others to have influenced behavior during the posttreatment choice test.

Amphetamine can engender state-dependent learning and induce interoceptive cues that can be conditioned as discriminative stimuli. Both drug effects would be expected to facilitate responding on the drug-assigned lever during periods of drug exposure (cf., Overton, 1974; Lal, 1977; Colpaert and Rosecrans, 1978; Goudie, 1991 for additional explanations). However, given that animals were drug-free during the choice test, it can be concluded that neither effect contributed positively to the greater preference of animals to respond on the drug-assigned lever during the choice test.

Owing to its primary rewarding properties, amphetamine could become associated with the drug-assigned stimuli and behavior, such that they would be more attractive and preferred during the choice test. Additionally or alternatively, amphetamine could engender a conditioned place preference for the area around the drug-assigned lever, consequently increasing the amount of time that animals spent near that area during the choice test and increasing the probability that animals would respond on the drugassigned lever. However, in Exp 2 animals did not exhibit an overall enhancement of instrumental responding on the drug-assigned lever. Therefore, it appears unlikely that the 
primary rewarding properties of amphetamine biased animals to respond on the drug-assigned lever.

It has been previously noted that presession injections of drug might lead to lasting changes in behavior conditioned during drug exposure by acutely altering performance and thereby indirectly impacting conditioning (eg Krivanek and McGaugh, 1969; McGaugh, 1973). This interpretation is potentially applicable to Exp 1, given that acute amphetamine exposure increased operant response rates during $\mathrm{S}+$ trials and thereby increased the number of rewards that animals earned. However, there was no evidence of an drug effect on performance in Exp 2, and the propensity of animals to respond on the drug-assigned lever during the choice test was quite similar between Exps 1 and 2. It is thus unlikely that amphetamine-induced performance effects on conditioning determined the pattern of responding during the post-treatment choice test.

It is possible that amphetamine influenced responding by acting directly on mechanisms that mediate or influence conditioning. Data of Exp 2 are consistent with this possibility. In Exp 2, there is evidence that the betweentest shift in responding was apparent during $\mathrm{S}+$ but not $\mathrm{S}-$ trials. This observation has been recently replicated in another experiment (unpublished observation). The $\mathrm{S}+$ specificity of the shift indicates that acute amphetamine actions impacted conditioning of the $\mathrm{S}+$, which more strongly controlled behavior during the choice test.

An increase in the strength of the $S+$ could be mediated by one or more drug effects on conditioning. For example, it could be mediated by a drug-induced facilitation of mechanisms that regulate the formation and strength of associations (eg stimulus-response, stimulus-reward associations) (Everitt and Robbins, 2005; Stein, 1964; Hill, 1970). Drug actions might also act on mechanisms that regulate the motivational properties of the S+ (Stewart, 1992; Robinson and Berridge, 1993, 2003). Moreover, as amphetamine metabolizes sufficiently slow as to be in brain up to several hours post-session (Lal and Feldmuller, 1975; Kuhn and Schanberg, 1978), it is possible that a drug effect on memory processes contributed to the relative increase in responding on the drug-assigned lever. The possibility of a drug effect on learning and memory processes, and the potential nature of that drug effect must be evaluated in future studies.

\section{Selective vs Non-Selective Effects of Amphetamine Exposure on Motivated Behavior}

As already described, the collective findings of numerous studies are consistent with the conclusion that lasting neuroadaptations induced by a history of psychomotor stimulant exposure can enhance various types of motivated behaviors, including those that have no history of having occurred in close temporal association with acute drug exposure. Nevertheless the findings of the present study provide novel evidence that a history of coincident amphetamine exposure, and conditioning, can enhance the expression of cue-controlled behaviors conditioned during drug exposure relative to similar behaviors conditioned in the absence of drug. This supports the broad interpretation that psychomotor stimulants can facilitate the subsequent expression of conditioned behavior and conditioned cue effects through multiple mechanisms, some of which are selective for behaviors and stimuli that occur during periods of acute drug actions and some are not.

\section{Neural Mechanisms That Potentially Mediated the Effect of the Amphetamine-Saline Treatment on Behavior during the Choice Test}

Based on neuropharmacological studies of various acute effects of psychomotor stimulants (Taylor and Robbins, 1984, 1986; Chu and Kelley, 1992; Kelley and Delfs, 1991; Wolterink et al, 1993), it is reasonable to hypothesize that acute effects of amphetamine on dopamine (DA) played a role in mediating the amphetamine-induced facilitation of the drug-assigned behavior/stimuli. A drug-induced increase in DA has been shown to modulate neuroplasticity mechanisms that normally underlie learning and memory, such as long-term potentiation and long-term depression (for a review, see Hyman and Malenka, 2001; Wolf, 2003; Wolf et al, 2004). It is possible that these activity-dependent effects of DA cause a selective strengthening of signaling of neurons that are engaged by behaviors/stimuli occurring during periods of drug exposure (Berke and Hyman, 2000; Peoples et al, 2004, 2006). However, given that acute exposure to amphetamine also elevates norepinephrine (NE) and serotonin (5-HT) and the effects of these monoamines on target neurons show activity-dependence, their potential role in mediating the selective facilitation of conditioned behavior by amphetamine should be considered as well.

\section{Implications for Drug Addiction}

A defining characteristic of drug addiction is that it is associated with an increase in drug-directed behaviors and a concomitant decrease in alternative motivated behaviors (American Psychiatric Association, 1994). Increases in drug seeking and taking are caused by drug-induced neuroadaptations (for reviews, see Hyman and Malenka, 2001; Everitt and Wolf, 2002; Kelley, 2004a; Robinson and Berridge, 1993, 2003; Kalivas et al, 2005; Nestler, 2005). However, the brain structures that mediate drug-directed behaviors (eg Wise and Bozarth, 1987; Robinson and Berridge, 1993; Koob et al, 1998; Koob, 1999; McFarland and Kalivas, 2001; Kelley, 2004a; Bossert et al, 2005) overlap with those that mediate other reward-directed behaviors (eg Everitt, 1990; Blackburn et al, 1992; Ikemoto and Panksepp, 1999; Cardinal et al, 2002; Kelley, 2004b; Schwienbacher et al, 2004; Wise, 2004, 2005; Salamone et al, 2005). It is currently unknown how neuroadaptations within the shared structures could contribute to a selective increase in drugdirected behaviors.

One possible explanation is that the co-occurrence of acute drug actions with drug-directed behaviors and drugassociated stimuli leads to a specific strengthening of the behaviors and/or the influence of the stimuli on behavior (Berke and Hyman, 2000; Peoples and Cavanaugh, 2003). This type of mechanism could potentially contribute to the differential changes in motivated behavior that define addiction. One basic prediction of this hypothesis is that the strength of conditioned behaviors and cue effects will be enhanced for behaviors and stimuli conditioned during 
acute drug actions relative to those conditioned during drug-free periods. The findings of the present study are consistent with this prediction.

\section{ACKNOWLEDGEMENTS}

We thank Drs Robert Rescorla and Daniel Gottlieb for helpful conversations related to the present studies and Jeffrey William for his assistance in data collection. This research was supported by NIDA DA05186 (Charles P O’Brien) and NIDA DA13401 (Laura L Peoples).

\section{REFERENCES}

American Psychiatric Association (1994). Diagnostic and Statistical Manual of Mental Disorders, 4th edn. American Psychiatric Association: Washington, DC.

Barr AM, Fiorino DF, Phillips AG (1999). Effects of withdrawal from an escalating dose schedule of D-amphetamine on sexual behavior in the male rat. Pharmacol Biochem Behav 64: 597-604.

Berke JD, Hyman SE (2000). Addiction, dopamine, and the molecular mechanisms of memory. Neuron 25: 515-532.

Blackburn JR, Pfaus JG, Phillips AG (1992). Dopamine functions in appetitive and defensive behaviors. Prog Neurobiol 39: 247-279.

Bossert JM, Ghitza UE, Lu L, Epstein DH, Shaham Y (2005). Neurobiology of relapse to heroin and cocaine seeking: an update and clinical implications. Eur J Pharmacol 526: 36-50.

Cardinal RN, Parkinson JA, Hall J, Everitt BJ (2002). Emotion and motivation: the role of the amygdala, ventral striatum, and prefrontal cortex. Neurosci Biobehav Rev 26: 321-352.

Chu B, Kelley AE (1992). Potentiation of reward-related responding by psychostimulant infusion into nucleus accumbens: role of dopamine receptor subtypes. Psychobiology 20: 153-162.

Colpaert FC, Rosecrans JA (1978). Stimulus Properties of Drugs: Ten Years of Progress. Elsevier/North Holland Biomedical Press: Amsterdam.

Everitt BJ (1990). Sexual motivation: a neural and behavioral analysis of the mechanisms underlying appetitive and copulatory responses of male rats. Neurosci Biobehav Rev 14: 217-232.

Everitt BJ, Dickinson A, Robbins TW (2001). The neuropsychological basis of addictive behavior. Brain Res Rev 36: 129-131.

Everitt BJ, Robbins TW (2005). Neural systems of reinforcement for drug addiction: from actions to habits to compulsion. Nat Neurosci 8: 1481-1489.

Everitt BJ, Wolf ME (2002). Psychomotor stimulant addiction: a neural systems perspective. J Neurosci 22: 3312-3320.

Fiorino DF, Phillips AG (1999). Facilitation of sexual behavior and enhanced dopamine efflux in the nucleus accumbens of male rats after d-amphetamine-induced behavioral sensitization. J Neurosci 19: 456-463.

Glick SD (1971). Facilitation or impairment of learning by $\mathrm{d}$-amphetamine as a function of stimuli. Psychopharmacologia (Berl) 21: 353-360.

Goudie AJ (1991). Discriminative stimulus properties of amphetamine, cathinone, and related agents. NIDA Res Monogr 116: 45-60.

Harmer CJ, Phillips GD (1998). Enhanced appetitive conditioning following repeated pretreatment with d-amphetamine. Behav Pharmacol 9: 299-308.

Harmer CJ, Phillips GD (1999). Enhanced conditioned inhibition following repeated pretreatment with d-amphetamine. Psychopharmacology 142: 120-131.

Hill RT (1970). Facilitation of conditioned reinforcement as a mechanism of psychomotor stimulation. In: Costa E, Garattini S (eds). Amphetamine and Related Compounds. Raven Press: New York. pp 781-795.
Hyman SE, Malenka RC (2001). Addiction and the brain: the neurobiology of compulsion and its persistence. Nat Rev Neurosci 2: 695-703.

Ikemoto S, Panksepp J (1999). The role of nucleus accumbens dopamine in motivated behavior: a unifying interpretation with special reference to reward-seeking. Brain Res Rev 31: 6-41.

Kalivas PW, Volkow N, Seamans J (2005). Unmanageable motivation in addiction: a pathology in prefrontal-accumbens glutamate transmission. Neuron 45: 647-650.

Kelley AE (2004a). Memory and addiction: shared neural circuitry and molecular mechanisms. Neuron 44: 161-179.

Kelley AE (2004b). Ventral striatal control of appetitive motivation: role in ingestive behavior and reward-related learning. Neurosci Biobehav Rev 27: 765-776.

Kelley AE, Delfs JM (1991). Dopamine and conditioned reinforcement. I. Differential effects of amphetamine microinjections into striatal subregions. Psychopharmacology 103: 187-196.

Killcross AS, Everitt BJ, Robbins TW (1997). Symmetrical effects of amphetamine and alpha-flupenthixol on conditioned punishment and conditioned reinforcement: contrasts with midazolam. Psychopharmacology 129: 141-152.

Koob GF (1999). The role of the striatopallidal and extended amygdala systems in drug addiction. Ann NY Acad Sci 877: 445-460.

Koob GF, Sanna PP, Bloom FE (1998). Neuroscience of addiction. Neuron 21: 467-476.

Krivanek JA, McGaugh JL (1969). Facilitating effects of pre- and post-trial amphetamine administration on discrimination learning in mice. Agents Actions 1: 36-42.

Kuhn CM, Schanberg SM (1978). Metabolism of amphetamine after acute and chronic administration to the rat. J Pharmacol Exp Ther 207: 544-554.

Kulkarni AS (1968). Facilitation of instrumental avoidance learning by amphetamine: an analysis. Psychopharmacologia 13: 418-425.

Lal H (1977). Discriminative Stimulus Properties of Drugs. Raven Press: NY.

Lal S, Feldmuller F (1975). Effect of amphetamine and apomorphine on brain monoamines and behavior in the immature and young adult rat. Arch Int Pharmacodyn Ther 218: 239-251.

Levens N, Akins CK (2004). Chronic cocaine pretreatment facilitates Pavlovian sexual conditioning in male Japanese quail. Pharmacol Biochem Behav 79: 451-457.

McFarland K, Kalivas PW (2001). The circuitry mediating cocaineinduced reinstatement of drug-seeking behavior. J Neurosci 21: 8655-8663.

McGaugh JL (1973). Drug facilitation of learning and memory. Annu Rev Pharmacol 13: 229-241.

Miller RR, Spear NE (1985). Information Processing in Animals: Conditioned Inhibition. Lawrence Erlbaum Associates: Hillsdale, NJ.

Nestler EJ (2005). Is there a common molecular pathway for addiction? Nat Neurosci 8: 1445-1449.

Nocjar C, Panksepp J (2002). Chronic intermittent amphetamine pretreatment enhances future appetitive behavior for drug- and natural-reward: interaction with environmental variables. Behav Brain Res 128: 189-203.

Olausson P, Jentsch JD, Taylor JR (2003). Repeated nicotine exposure enhances reward-related learning in the rat. Neuropsychopharmacology 28: 1264-1271.

Overton DA (1974). Experimental methods for the study of statedependent learning. Fed Proc 33: 1800-1813.

Peoples LL, Cavanaugh D (2003). Differential changes in signal and background firing of accumbal neurons during cocaine selfadministration. J Neurophysiol 90: 993-1010.

Peoples LL, Kravitz AV, Lynch KG, Cavanaugh DJ (2006). Accumbal neurons that are activated during cocaine self- 
administration are spared from inhibitory effects of repeated cocaine self-administration. Neuropsychopharmacology, print copy in press (originally published online August 4, 2006, at www.acnp.org/citations/Npp080406060173/).

Peoples LL, Lynch KG, Lesnock J, Gangadhar N (2004). Accumbal neural responses during the initiation and maintenance of intravenous cocaine self-administration. J Neurophysiol 91: 314-323.

Robbins TW (1975). The potentiation of conditioned reinforcement by psychomotor stimulant drugs: a test of Hill's hypothesis. Psychopharmacologia (Berl) 45: 103-114.

Robbins TW (1978). The acquisition of responding with conditioned reinforcement: effects of pipradrol, methylphenidate, d-amphetamine and nomifensine. Psychopharmcology 58: 79-87.

Robbins TW, Watson BA, Gaskin M, Ennis C (1983). Contrasting interactions of pipradrol, d-amphetamine, cocaine, cocaine analogues, apomorphine and other drugs with conditioned reinforcement. Psychopharmacology 80: 113-119.

Robinson TE, Berridge KC (1993). The neural basis of drug craving: an incentive-sensitization theory of addiction. Brain Res Rev 18: 247-291.

Robinson TE, Berridge KC (2003). Addiction. Annu Rev Psychol 54: 25-53.

Salamone JD, Correa M, Mingote SM, Weber SM (2005). Beyond the reward hypothesis: alternative functions of nucleus accumbens dopamine. Curr Opin Pharmacol 5: 34-41.

Schwienbacher I, Fendt M, Richardson R, Schnitzler H-U (2004). Temporary inactivation of the nucleus accumbens disrupts acquisition and expression of fear-potentiated startle in rats. Brain Res 1027: 87-93.

Stein L (1964). Amphetamine and neural reward mechanisms. In: Steinberg H, de Reuck AA, Knight J (eds). Animal Behavior and Drug Action. Churchill: London. pp 91-118.

Stewart J (1992). Neurobiology of conditioning to drugs of abuse. Ann NY Acad Sci 654: 335-346.

Taylor JR, Horger BA (1999). Enhanced responding for conditioned reward produced by intra-accumbens amphetamine is potentiated after cocaine sensitization. Psychopharmacology 142: $31-40$.
Taylor JR, Jentsch JD (2001). Repeated intermittent administration of psychomotor stimulant drugs alters the acquisition of Pavlovian approach behavior in rats: differential effects of cocaine, d-amphetamine and 3, 4-methylenedioxymethamphetamine ('ecstasy'). Biol Psychiatry 50: 137-143.

Taylor JR, Robbins TW (1984). Enhanced behavioral control by conditioned reinforcers following microinjections of d-amphetamine into the nucleus accumbens. Psychopharmacology 84: 405-412.

Taylor JR, Robbins TW (1986). 6-Hydroxydopamine lesions of the nucleus accumbens, but not of the caudate nucleus, attenuate enhanced responding with reward-related stimuli produced by intra-accumbens d-amphetamine. Psychopharmacology 90: 390-397.

White NM (1996). Addictive drugs as reinforcers: multiple partial actions on memory systems. Addiction 91: 921-949.

Wise RA (2004). Drive, incentive, and reinforcement: the antecedents and consequences of motivation. Nebr Symp Motiv 50: $159-195$

Wise RA (2005). Forebrain substrates of reward and motivation. J Comp Neurol 493: 115-121.

Wise RA, Bozarth MA (1987). A psychomotor stimulant theory of addiction. Psychol Rev 94: 469-492.

Wolf ME (2003). LTP may trigger addiction. Mol Interv 3: 248-252.

Wolf ME, Sun X, Mangiavacchi S, Chao SZ (2004). Psychomotor stimulants and neuronal plasticity. Neuropharmacology 47(Suppl 1): 61-79.

Wolterink G, Phillips G, Cador M, Donselaar-Wolterink I, Robbins TW, Everitt BJ (1993). Relative roles of ventral striatal d1 and d2 dopamine-receptors in responding with conditioned reinforcement. Psychopharmacology 110: 355-364.

Wyvell CL, Berridge KC (2000). Intra-accumbens amphetamine increases the conditioned incentive salience of sucrose reward: enhancement of reward 'wanting' without enhanced 'liking' or response reinforcement. J Neurosci 20: 8122-8130.

Wyvell CL, Berridge KC (2001). Incentive sensitization by previous amphetamine exposure: increased cue-triggered 'wanting' for sucrose reward. J Neurosci 21: 7831-7840. 